\title{
Auf dem Weg zu Professionalisierung
}

\author{
Aufgaben der Pflegewissenschaft im Zentrum der procareScience Lecture
}

Pflegetheorien ohne Pflegeerfahrung sind leer. Umgekehrt helfen Theorien dabei, Fragen der Praxis zu beantworten, weil sie Merkmale und Zusammenhänge auf eine breiter gültige Ebene stellen, hinterfragen und überprüfen. Pflege braucht Theorien, um die Zusammenhänge pflegerischen Handelns erkennen und ihr Wissen systematisch entfalten zu können.

In der diesjährigen procareScience-Lecture beim PflegeManagementForum Anfang März in Wien illustrierte Dr. Eleonore Kemetmüller vom Department für Pflegewissenschaft der NÖGUS, wie vielfältig diese Zusammenhänge sind. Und sie zeigte die vielschichtigen Rahmenbedingungen und Ereignisse auf, die dazu geführt haben, dass dieses Department per März 2016 in die Karl Landsteiner Privatuniversität - als Fachbereich Pflegewissenschaft - mit Standort Campus Krems eingegliedert wurde.

Die Akademisierung der Pflege stellt die Grundlage für die Professionalisierung dar. Denn die Theorien tragen dazu bei, Zusammenhänge als solche zu erkennen und daraus Konsequenzen im Handeln ziehen zu können. Ebenso muss sich die Praxis immer wieder hinterfragen. „Im Kontext der professionellen pflegerischen Arbeit“, so Kemetmüller, „ist ein theoretischer Fundus ebenso unabdingbar wie eine sich reflektierende Praxis." Gerade in der Pflege ist dies mit ganz spezifischen Problemen verbunden, da im pflegerischen Handeln eine Vielzahl an Komponenten wirkt und wirksame Interventionen sich oft nicht auf Einzelfaktoren zurückführen lassen. Gleichzeitig ist Pflege zu einem großen Anteil Beziehungsarbeit, die sich schwer messen lässt. In Niederösterreich wurde mit der Einrichtung eines Departments für Pflegewissenschaft im NÖGUS - dem Niederösterreichischen Gesundheits- und Sozialfonds - vor vier Jahren ein Pionierschritt getan, wie Kemetmüller berichtete.

\section{„Wir konnten uns neu erfinden!“}

Die überraschende Schließung der Diplomausbildung am Landesklinikum Krems 2012 ermöglichte die Entwicklung eines

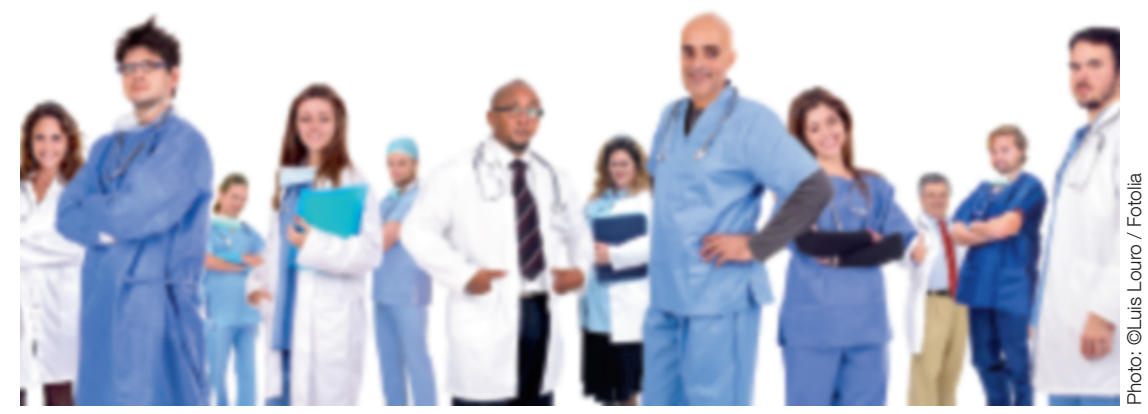

Wissenschaft unterstützt die pflege auf dem Weg zur Professionalisierung.

Departments für Pflegewissenschaft, das als Beratungs- und Forschungsstelle für Pflegeberufe dem NÖGUS zugehörig war. Hochqualifizierte pflegewissenschaftliche Mitarbeiterinnen standen also zur Verfügung, ebenso eine starke Motivation zur Entwicklung von Zukunftsperspektiven und „Mitstreiter und Fürsprecher“. „Wir konnten uns selbst neu erfinden“, so Kemetmüller zur Ausgangssituation, die von unklaren Auftragsformulierungen und geringen Ressourcen geprägt war. Als eigenen Auftrag definierte man also Kundenorientierung in Form von Beratung in einer dem stetigen Wandel unterworfenen Praxisentwicklung.

Mittlerweile wurden mehrere Projekte abgeschlossen, wie z.B.:

- Pflegerelevante Literaturdatenbanken in NÖ Gesundheits- und Krankenpflegeschulen

- Eine Entscheidungshilfe für Pflegeklassifikationssysteme

- Ein Praxiskonzept für Palliativpflege

- Die Begleitung bei der Umsetzung des Pflegemodells nach Roper - Logan Tierney

Laufende Projekte beschäftigen sich mit den Themenschwerpunkten Ethik und Evidence Based Nursing (EBN). Projektziel für den Bereich Ethik ist die Erarbeitung eines Konzeptes zum Thema „ethische Entscheidungsfindungen im NÖ Gesundheitswesen." Das Pflegeinformationszentrum „Florence“ mit den Teilbereichen Recherche und Anfrage Evidence Based Nursing ist der zweite wichtige Bestandteil des Leistungsspektrums. Die Namensgebung bezieht sich auf die hochgebildete Engländerin Florence Nightingale, die Wegbereiterin einer professionellen Krankenpflege und Vorbild für Generatio- nen von Pflegepersonen. Mit der umfangreich vernetzten Sammlung „Florence-Recherche" bietet man den Einstieg in die pflegerelevante Recherche in Journalen, Datenbanken und bei Websites nationaler und internationaler Organisationen und Einrichtungen, um Evidence Based Nursing zu unterstützen.

Das Ziel des Florence Anfrageservices EBN liegt in einer Qualitätsentwicklung und -sicherung der pflegerischen Versorgung durch die Förderung einer evidence-basierten Pflegepraxis. Neueste wissenschaftlich belegte Erkenntnisse im Verantwortungsbereich der Pflege sollen die Entscheidungsfindung der Pflegepersonen und Patienten leiten, so Kemetmüller.

Seit März 2016 ist das Department für Pflegewissenschaft des NÖGUS nun als Fachbereich Pflegewissenschaft in der Karl Landsteiner Universität am Standort Campus Krems eingegliedert. Als Aufgaben sieht man neben den Schwerpunkten „Klinische Ethikberatung“ und „Pflegeinformationszentrum Florence“, auch Grundlagenforschung und klinische Forschung mit dem Schwerpunkt „Pflege“, der Consultingleistungen für Dritte, der Durchführung von wissenschaftlichen Symposien und Fachvorträgen, sowie $\mathrm{Pu}$ blikationen in Fachjournalen. „Erst wenn akademisches Personal in der Praxis akzeptiert wird, die Pflege auf wissenschaftlich überprüfter Evidenz basiert und sich als eigenständige Disziplin präsentiert, ist eine Professionalisierung erreicht", stellte Kemetmüller fest.

Quelle: procareScience-Lecture von Eleonore Kemetmüller: „Theorie ohne Praxis ist leer, Praxis ohne Theorie ist blind." Beim Pflege Management Forum 2016, 4. März 2016, Wien 